\title{
Analysis of the Effective Ingredients in Tea
}

\author{
Yanjun Liu ${ }^{1}$ \\ ${ }^{1}$ Chongqing No.1 International Studies School, Chongqing, China, 401120 \\ *Corresponding author. Email:1139407809@qq.com
}

\begin{abstract}
Through the determination of the active ingredients of tea and the study of the effect of tea making on its content, it has been proved that tea is indeed beneficial to the health of human body. Therefore, in this paper, the extraction and improvement of active ingredients are studied, and the application of active ingredients in human body is also analyzed in order to provide suggestions for future investigation. Finally, the improvement on proportion of active ingredients are shown to produce effect with the change in process of making tea.
\end{abstract}

Keywords: Tea polyphenols, Caffeine, L-theanine, Extract method, Usage and outlook

\section{INTRODUCTION}

Nowadays, the word "regimen", which means a continuous process to pay attention to medication, exercise and diet, or other methods in order to improve individual's physical conditions, is being taken seriously. Tea, which is one of the most representative healthy drinks, is always mentioned about regimen. Thus it is common to see that the useful components in tea are referred in some articles about regimen like curing cancers, lower cholesterol and blood pressure. To prove whether it is true that the previous studies have talked about the function of tea, the analysis of useful components in tea is essential. In addition, the left effect produced by processing tea before selling is also included. Besides, the method of extraction and its improvement and medical use in these components are also considered.

\section{ANALYSIS OF COMPONENTS}

Tea, as one of the drinks that can improve physical conditions, contains more than 700 known chemical compositions, which also causes fragrance and complex taste when people drink it. Among these chemicals, tea polyphenols, alkaloids (mainly caffeine), and amino acids (mainly L-theanine) are the most important parts of tea, which are also known as characteristic components in tea.

\subsection{Caffeine}

1,3,7-Trimethylxanthine $\left(\mathrm{C}_{8} \mathrm{H}_{10} \mathrm{~N}_{4} \mathrm{O}_{2}\right)$, known as caffeine, is a white needle-shaped crystal. It is a central nerve stimulant that temporarily beats ongoing sleepiness and restores energy, clinically used to treat neurasthenia and coma resuscitation. In addition, caffeine is the most commonly used psychotropic drug in the world. Tea is an important source of caffeine, and the caffeine content of each cup of tea is generally only half that of each cup of coffee. Specific varieties of tea, such as black tea and oolong tea, contain more caffeine than other kinds of teas. Tea contains a small amount of cocoa base and a slightly higher soybase than coffee. Caffeine has huge effect in the excitable cerebral cortical layer, which can achieve the purpose of godlising. In addition, caffeine not only has some effects on blood vessel contraction and on fatigue caused by tension headaches, but also has an improved effect, which is the most common effect in tea leaves. In the compound preparation of cold medicine, caffeine can reduce the drowsiness caused by ammonia benzene sensitivity adverse reactions, as well as strengthen the role of some analgesics in the central nervous system. It can be seen that the application is relatively widespread. However, the color of tea can hardly indicate the amount of caffeine. The caffeine content in Japanese green tea is much lower than that of many black teas, such as Masayama' small-bred tea, which is almost decaffeinated. Normally, the proportion of caffeine in dried tea is $2 \%$ to $4 \%$. Due to the harm to human beings of excessive intake, especially the addiction, caffeine content is also an important quality index of tea, which could influenced by the process of dying tea. Professor. Xiang Leiwen, work in Fuqing Branch of Fujian Normal University in China, said that during the process, the chemical composition in tea is not completely isolated. Chemical reactions may occur between the chemical compositions, and the reaction 
speed should be accelerated as the temperature rises. In the process of first three steps, because the tea cells are not seriously damaged, the various substances in the tea cells are not completely dissolved, and the temperature is low or at high temperatures in a short time, so the effect is not obvious. However, during kneading and molding, tea cells are squeezed, and cells are broken. Cytetons flow out and remain on the tea leaves, which facilitates chemical reactions between substances, coupled with higher temperatures during the drying process, resulting in an increased reaction, reflected in a significant increase in caffeine proportion [1].

\subsection{L-theanine}

L-theanine is a unique free amino acid in tea, which occupies over $50 \%$ of total amino acid. Figure 1 shows the chemical structure of L-theanine. It is also the main compounds to reduce the bitter taste in the tea by enhancing sweetness. Theanine also has a good antifatigue and calming effect. As it is known that after intaking drinks with caffeine, people will feel excited; while this phenomenon cannot happen to tea, which also has caffeine. By contrast, people feel very calm, comfortable, relaxed and have a smooth mood after drinking tea. By comparing the different reactions of the body with caffeine intake by drinking tea as well as directly ingesting the same amount of caffeine, it can be proved that it is resulted from the effect of L-theanine. Like the caffeine, the process also has effect on the proportion of L-theanine in tea. For example, in the processing of $\mathrm{Pu}$-erh tea, the content of free amino acids dropped significantly, mainly in the original tea to fermentation semi-finished processing stage. Due to fermentation, a large number of microorganisms need to consume a huge amount of amino acids as nitrogen sources, resulting in a reduction in amino acid content. In addition, at the end of the pile fermentation, drying must be done in order to prevent excessive pile. During dying, due to the high temperature, some amino acids have volatile loss, while other amino acid contain deoxygenation to form amines or lost by releasing ammonia or carbon dioxide. As a result, the proportion of amino acid significantly decreases [2].

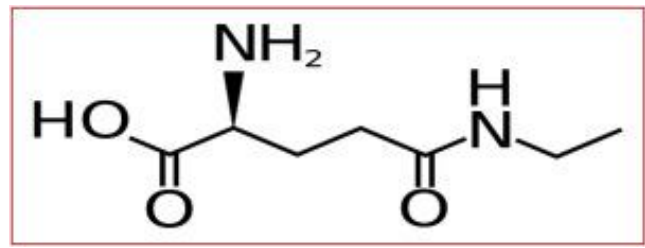

Figure 1 Chemical structure of L-theanine

\subsection{Tea Polyphenols}

Tea polyphenols are general called polyphenols in tea, normally contain $20-30 \%$ in total mass of tea leaves. It is often regarded as deodorization in daily life like in chewing gum to suppress bad breath. In tea leaves, it provides the color and taste and brings antioxidation to human beings after drinking. And how it can be affected by the process? Research shows that in the process of tanning tea to fermentation to semi-finished products, the taste of tea will gradually change from bitter taste to $\mathrm{Pu}-$ erh tea's unique quality. The water-soluble transformation of polyphenols (theabrowmine) is the main body of the shape and soup colors that make up tea, which also leads to a decrease in tea polyphenols after fermentation [2]. Besides the Pu-erh tea, black tea also has a decrease in tea polyphenol during fermentation in order to produce a strong taste of sweetness.

The following figure 2 shows the major tea polyphenols. Tea polyphenols can protect cells from oxidative damage by regulating certain cell signaling pathways. In addition, figure 3 shows the cellular pathways regulated by tea polyphenols during their antioxidant activity. Therefore, tea polyphenols is beneficial to human health, which might be a good choice for people to prevent aging and keep a young condition.

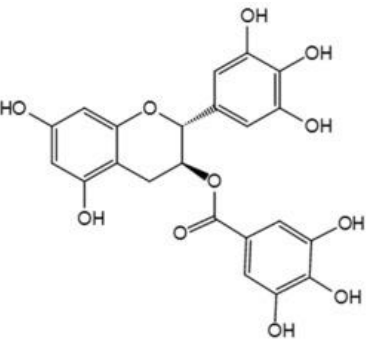

$(-)$-epigallocatechin-3-galate (EGCG)<smiles>CCOc1cc(O)c2c(c1)OC(c1cc(O)c(O)c(O)c1)C(O)C2</smiles><smiles>O=C(O[C@H]1Cc2c(O)cc(O)cc2OC1c1ccc(O)c(O)c1)c1cc(O)c(O)c(O)c1</smiles>

$(-)$-epicatechin-3-gallate (ECG)<smiles>Oc1cc(O)c2c(c1)OC(c1ccc(O)c(O)c1)C(O)C2</smiles>

Figure 2 Structures of the major tea polyphenols [11]

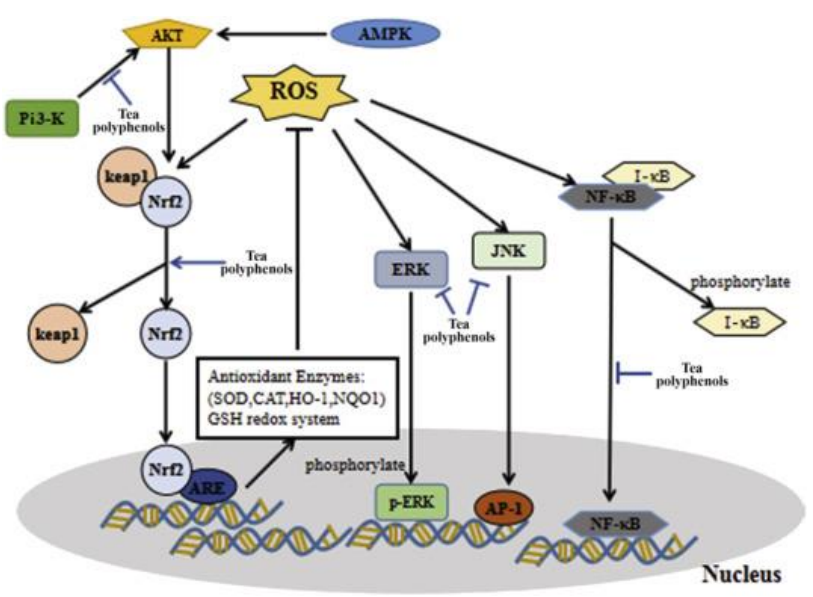

Figure 3 The cellular pathways regulated by tea polyphenols during their antioxidant activity [11] 


\section{DISCUSSION}

All the useful compound mentioned are affected by processing to certain degree, so whether the compound still work in human beings after being changed in proportion of the useful components?

There are always four steps in the traditional processing of making tea [4]. At first, fresh tea is picked, tiled on a shelf with $2-3 \mathrm{~cm}$ thick. These leaves would be placed in the natural environment, so that the tea leaves exude some moisture and become wilted. Next, the tea leaves would be stirred and fried in a machine of about $300{ }^{\circ} \mathrm{C}$ for $20-50$ minutes. After the tea leaves are cooled, they are put in a kneading machine and kneaded in a lightto-heavy manner. Finally, the tea leaves are dried with the temperature controlled to $120-130{ }^{\circ} \mathrm{C}$ and tea content about $20 \%-25 \%$ [6].

By contrast, the wilting and kneading do not affect the components in tea [3]. However, the frying and drying process have the influence with high temperature to decompose the component in tea leaves. As a result, the proportion of the main three components decreases by 10 $15 \%$ during the process. Besides, in different types of tea, brewing time can also cause changes in these substances. Research shows the best brewing time for black tea is 30s-1min, green tea should be brewed 3-10 minutes, brewing up to four times, with 2-3 times as the best. The tea should be drunk after brewing immediately in order to prevent the decrease in components [5].

For the extraction of these effective substances, there are generally three methods - organic solvent extraction, ion precipitation extraction and column analysis. Organic solution extraction method is obtained from ethyl acetate extraction after decolorization and de-coffee base of tea liquid by organic solvents [7]. This method has the disadvantage that the process is cumbersome and complex with the distillation for several times. In addition, it uses toxic organic solvents such as chloroform, making the product and operation less safe. To improve it, the efficiency is the most considered with the less operation and time needed and the avoid for using toxic solvent.

Ion precipitation extraction is to separate the sediment by precipitation agent after adjusting the $\mathrm{pH}$ of the tea [8], and then $40 \%$ sulfuric acid solution is added to destroy the sediment, so that the extracted compound is transferred to the solution. Finally, the components would be extracted by ethyl acetate. However, this approach has more stringent requirements for the $\mathrm{pH}$ of the liquid with 5.5-6, because in this $\mathrm{pH}$ value, the effective molecules easily and metal ions produce precipitation, as a result of easier extraction. Higher or lower $\mathrm{pH}$ can lead to oxidation of active components. Therefore, ion precipitation extraction need expand the range of $\mathrm{pH}$ value for more effective extraction.
Column analysis method uses different ion exchange resins on tea polyphenols and coffee base adsorption properties to separate the preparation of tea polyphenols. This method is available with simple operation and less energy consumption, but the used separation column would have a higher selectivity and a large equilibrium adsorption capacity of tea polyphenols.

Recently, animal experiments and population studies have suggested that tea or tea extract can inhibit the formation and development of a variety of tumors. As one of the main causes of esophageal and stomach cancers, nitro compounds can be inhibited by tea polyphenols at low concentrations in order to resist the nitrosation [9]. In addition, tea polyphenols can also fight cancer, but its anti-cancer rationale is not yet clear. There is a widespread speculation that it may be related to inducing apoptosis to inhibit cell proliferation. Besides it, it also has been shown that the L-theanine can help suppress tumors by improving the efficacy of a variety of antitumor drugs. Adri-amycin, for example, inhibits some tumors. By using it with theanine, the oozing of amycin from tumor cells can be reduced, resulting in a 2.1 -fold increase in growth inhibition of living tumors [10].

\section{CONCLUSION}

The content of tea polyphenols in tea decreases with the increase of fermentation. The content of caffeine increased significantly while the content of theanine decreases significantly after fermentation. The three components truly have the effect to improve well-beings, but the long-term use should be essential with correct brewing time. However, the method of extraction of useful components should be improved and the problems in application should also be addressed. As potential anticancer components, more studies in tea polyphenols and theanine are needed, in order to find the theory of anticancer rationale and efficient combination with other anti-cancer drugs for further application.

\section{ACKNOWLEDGMENTS}

First and foremost, my deepest gratitude would be shown to my teachers and professors with valuable guidance in every steps of the writing in this thesis. Further, I would like to thank my parents and friends for their encouragement and support during my writing. Without all their help and support, I could not completes my paper.

\section{REFERENCES}

[1] Xiang Leiwen, The effect of the production process on the caffeine content of oolong tea, Amino acids and biological resources 2009 No. 03, pp. 15-17.

[2] Chen Xiaoqiang, Ye Yang, Cheng Hao, Yin Junfeng, Sun Cheng, Comparative Analysis of 
Theanine, Caffein and Polyphenolic Constituents in Green Tea, Black Tea and Puer Tea, Food Research and Development 12, 2007, pp.141-144.

[3] Xu Mingzhu, Chen Yuqiong, The influence of processing process on the quality of black tea and the application of new technology, China Tea Processing 04, 2010, pp. 33-35.

[4] Tian Yang, Xiao Rong, Xu Kunlong, Jiang Bo, Shi Chongying, Changes and Correlations of Main Chemical Components during Pu'er Tea Processing, Food Science 11, 2010, pp. 20-24.

[5] Luo Jing, Gu Yingying, Liu Yicheng, Guo Liang, Analysis of the Polyphenols and Caffeine in Different Teas, Guizhou Tea 2013 No. 01, pp. 1015.

[6] Huang Shaoman, Feng Wanyin, Li Hongyan, The influence of different tea-making processes and times on the polyphenol content of tea in tea was studied, Beverage Industry 2013 No. 03, pp.26-28.

[7] Dong Wenbing, Hu Ying, The preparation process and application of tea polyphenols, Northwest Journal of Light Industry [J], 2002, 20( 4): 18-24.

[8] Yu Zhaoxiang, Wang Xiaoping, Study on the extraction of tea polyphenols from compound sediments [J].Food industry technology, 2001, 22( 3), pp. 32-34.

[9] RU AN Dongliang, ZHANG Yingfeng ,WANG Fengling, LI Changjiang, LIU Ping, ZHENG Xiangmei, ZHENG Xiangjun, Extraction and application of tea polyphenols, Journal of Bohai University (Natural Sciences Edition) 2007 No. 01, pp. 6-11.

[10] Zhu Song, Wang Hongxin, Physiological function of theanine and its application in food, Food Research and Development, June 2005 Volume 26, No. 3, pp. 18-20.

[11] Zhaoming Yan, Yinzhao Zhong, Yehui Duan, Qinghua Chen, Fengna Li, Antioxidant mechanism of tea polyphenols and its impact on health benefits, Animal Nutrition, Vol. 6, Issue 2, 2020, pp. 115-123. 